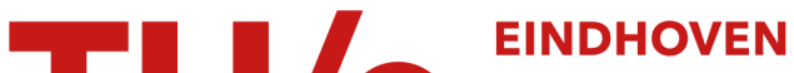 \\ UNIVERSITY OF \\ TECHNOLOGY
}

\section{Some peculiarities of exponential random variables}

Citation for published version (APA):

Litvak, N. (2000). Some peculiarities of exponential random variables. (Report Eurandom; Vol. 2000039).

Eurandom.

Document status and date:

Published: 01/01/2000

\section{Document Version:}

Publisher's PDF, also known as Version of Record (includes final page, issue and volume numbers)

\section{Please check the document version of this publication:}

- A submitted manuscript is the version of the article upon submission and before peer-review. There can be important differences between the submitted version and the official published version of record. People interested in the research are advised to contact the author for the final version of the publication, or visit the $\mathrm{DOI}$ to the publisher's website.

- The final author version and the galley proof are versions of the publication after peer review.

- The final published version features the final layout of the paper including the volume, issue and page numbers.

Link to publication

\section{General rights}

Copyright and moral rights for the publications made accessible in the public portal are retained by the authors and/or other copyright owners and it is a condition of accessing publications that users recognise and abide by the legal requirements associated with these rights.

- Users may download and print one copy of any publication from the public portal for the purpose of private study or research.

- You may not further distribute the material or use it for any profit-making activity or commercial gain

- You may freely distribute the URL identifying the publication in the public portal.

If the publication is distributed under the terms of Article 25fa of the Dutch Copyright Act, indicated by the "Taverne" license above, please follow below link for the End User Agreement:

www.tue.nl/taverne

Take down policy

If you believe that this document breaches copyright please contact us at:

openaccess@tue.nl

providing details and we will investigate your claim. 


\title{
Some peculiarities of exponential random variables
}

\author{
Nelly Litvak
}

November 23, 2000

\begin{abstract}
Consider a non-random function whose arguments are i.i.d. exponential random variables. What will be a conditional distribution of this function given that the arguments satisfy a certain linear inequality? In this paper we describe a method which sometimes allows answering this question. We present two examples where our method leads to unexpectedly simple answers. We give an interpretation of the results via the travel time in carousel systems.
\end{abstract}

\section{Introduction}

We start with introducing some notations which are used throughout the paper. Let $X_{1}, X_{2}, \ldots$ be i.i.d. exponential r.v.'s with mean 1. Denote

$$
S_{0}=0 ; \quad S_{i}=\sum_{j=1}^{i} X_{j}, \quad i \geq 1 .
$$

Further, let $U_{1}, U_{2}, \ldots, U_{n}$ be independent random variables uniformly distributed on the interval $[0,1)$. Let $U_{(1)}, U_{(2)}, \ldots, U_{(n)}$ denote the order statistics of $U_{1}, U_{2}, \ldots, U_{n}$. Put $U_{(0)}=0, U_{(n+1)}=1$. We shall define uniform $(n+1)$-spacings $D_{1}, D_{2}, \ldots, D_{n+1}$ as

$$
D_{i}=U_{(i)}-U_{(i-1)}, \quad 1 \leq i \leq n+1
$$

and we shall denote

$$
\tilde{S}_{0}=0 ; \quad \tilde{S}_{i}=\sum_{j=1}^{i} D_{j}, \quad i \geq 1 .
$$

The well-known property of spacings (cf. Pyke [8], [9]) is that they are distributed as i.i.d. exponentials divided by their sum:

$$
\left(D_{1}, D_{2}, \ldots, D_{n+1}\right) \stackrel{d}{=}\left(X_{1} / S_{n+1}, X_{2} / S_{n+1}, \ldots, X_{n+1} / S_{n+1}\right) .
$$

Here and below we use a common notation

$$
X \stackrel{d}{=} Y
$$


to indicate that random variables $X$ and $Y$ have the same distribution.

In their paper [7] Litvak and Adan proved that

$$
\sum_{i=1}^{n} \min \left\{\tilde{S}_{i}, D_{i+1}\right\} \stackrel{d}{=} \sum_{i=1}^{n}\left(1-\frac{1}{2^{i}}\right) D_{i} .
$$

In order to do it they elaborated an approach which was presented for the first time in Litvak et al. [6].

Remark 1.1 As we shall see below from Remark 3.2 the equation

$$
\sum_{i=1}^{n} \min \left\{S_{i}, X_{i+1}\right\} \stackrel{d}{=} \sum_{i=1}^{n}\left(1-\frac{1}{2^{i}}\right) X_{i}
$$

can be proved along the same lines as (3). Note that (2) is not sufficient to prove that (3) and (4) are equivalent, because in general none of the equalities

$$
f\left(X_{1}, \ldots, X_{n+1}\right) \stackrel{d}{=} g\left(X_{1}, \ldots, X_{n+1}\right)
$$

and

$$
f\left(X_{1}, \ldots, X_{n+1}\right) / S_{n+1} \stackrel{d}{=} g\left(X_{1}, \ldots, X_{n+1}\right) / S_{n+1}
$$

implies the other. For example, let $F(x)$ be a distribution function of $S_{n+1}$. Since a random variable $1-e^{-X_{1}}$ is uniformly distributed on $[0,1)$ we have

$$
F^{-1}\left(1-e^{-X_{1}}\right) \stackrel{d}{=} S_{n+1} \text {. }
$$

However,

$$
F^{-1}\left(1-e^{-X_{1}}\right) / S_{n+1} \stackrel{d}{\neq} S_{n+1} / S_{n+1} \equiv 1 .
$$

Nevertheless, let us show that (3) and (4) are indeed equivalent. Denoting

$$
V_{n}=\sum_{i=1}^{n} \frac{\min \left\{S_{i}, X_{i+1}\right\}}{S_{n+1}}, \quad W_{n}=\sum_{i=1}^{n}\left(1-\frac{1}{2^{i}}\right) \frac{X_{i}}{S_{n+1}},
$$

we can rewrite (4) as

$$
S_{n+1} V_{n} \stackrel{d}{=} S_{n+1} W_{n}
$$

Also, using (2) equation (3) can be rewritten as

$$
V_{n} \stackrel{d}{=} W_{n} .
$$

Note that $V_{n}$ and $W_{n}$ are functions of the normalized exponentials $X_{1} / S_{n+1}, X_{2} / S_{n+1}, \ldots$, $X_{n+1} / S_{n+1}$, which are independent of $S_{n+1}$ (cf. Pyke [8]). Hence, (5) follows from (6). Furthermore, since all moments of $S_{n+1}, V_{n}$ and $W_{n}$ exist, it follows from (5) that

$$
E\left(V_{n}\right)^{k}=E\left(W_{n}\right)^{k}, \quad k \geq 1 .
$$

Clearly, each of the random variables $V_{n}$ and $W_{n}$ has a distribution which is concentrated on a closed interval. Such a distribution is uniquely defined by its moments, because its Laplace transform is analytic on the whole complex plane (see also Section VII.3 and Section XV.4 of Feller [4]). From the above we conclude that (5) and (6) are equivalent. 
In Section 2 of this paper we generalize the approach from Litvak et al. [6], Litvak and Adan [7], and we point out its most important properties. In Section 3 we give two examples where the generalized approach leads to nice results. The first example implies (3) and (4) as special cases.

Section 4 concerns an application of the results to the travel time in carousel systems. A carousel (or paternoster) is an automated warehousing system consisting of a large number of shelves and drawers rotating in a closed loop in either direction. The locations of required items are uniformly distributed on a circle. Thus, the distances between adjacent items are uniform spacings.

Formula (3) was obtained in Litvak and Adan [7] while considering the travel time in carousel systems under the Nearest Item (NI) heuristic, when the next item to be picked is always the nearest to the picker's current position. The second example from Section 3 allows obtaining a distribution of the shortest travel time when the picker may perform not more than a given number of steps, say, counterclockwise, and after that he must pick the rest of the items clockwise. We study this kind of routes in detail and compare the best of them with the NI heuristic.

\section{Conditioning on linear inequalities}

In this section we explain our arguments for conditioning on linear inequalities. Denote

$$
\mathbf{c}=\left(c_{1}, c_{2}, \ldots\right), \quad c_{1}, c_{2}, \ldots>0
$$

and

$$
S_{0}(\mathbf{c})=0 ; \quad S_{i}(\mathbf{c})=\sum_{j=1}^{i} c_{j} X_{j}, \quad i \geq 1 .
$$

We consider random events

$$
E_{i+1, k}(\mathbf{c})=\left[S_{k-1}(\mathbf{c})<X_{i+1}<S_{k}(\mathbf{c})\right], \quad 1 \leq k \leq i ; \quad E_{i+1, i+1}(\mathbf{c})=\left[S_{i}(\mathbf{c})<X_{i+1}\right] .
$$

Note that only the first $i$ coordinates of vector $\mathbf{c}$ play a role in $E_{i+1, k}(\mathbf{c})$.

Litvak et al. [6] as wel as Litvak and Adan [7] only considered the case $c_{1}=c_{2}=\cdots=1$. However, their arguments can be generalized for arbitrary positive $c_{j}$ 's.

Given an event $E_{i+1, k}(\mathbf{c})$ for some $k=2,3, \ldots, i$, the random variable $c_{1} X_{1}$ is the minimum of $c_{1} X_{1}$ and $X_{i+1}$, and thus it is exponential with mean $c_{1} /\left(c_{1}+1\right.$ ) (see also Figure 1). Due to the memory-less property the overshoot of $X_{i+1}$ is again exponential with mean 1 . Hence, we can repeat the argument for $c_{2} X_{2}$ and so on. Eventually random variable $X_{i+1}-S_{k-1}(\mathbf{c})$ is less than $c_{k} X_{k}$, so it is exponential with mean $c_{k} /\left(c_{k}+1\right)$, and the overshoot of $c_{k} X_{k}$ is exponential with mean $c_{k}$. Since the event $E_{i+1, k}(\mathbf{c})$ does not provide any information on the other random variables, their distribution remains the same. Hence, assuming $E_{i+1, k}(\mathbf{c})$ the random variables $X_{1}, X_{2}, \ldots$ can be replaced by i.i.d. with mean 1 


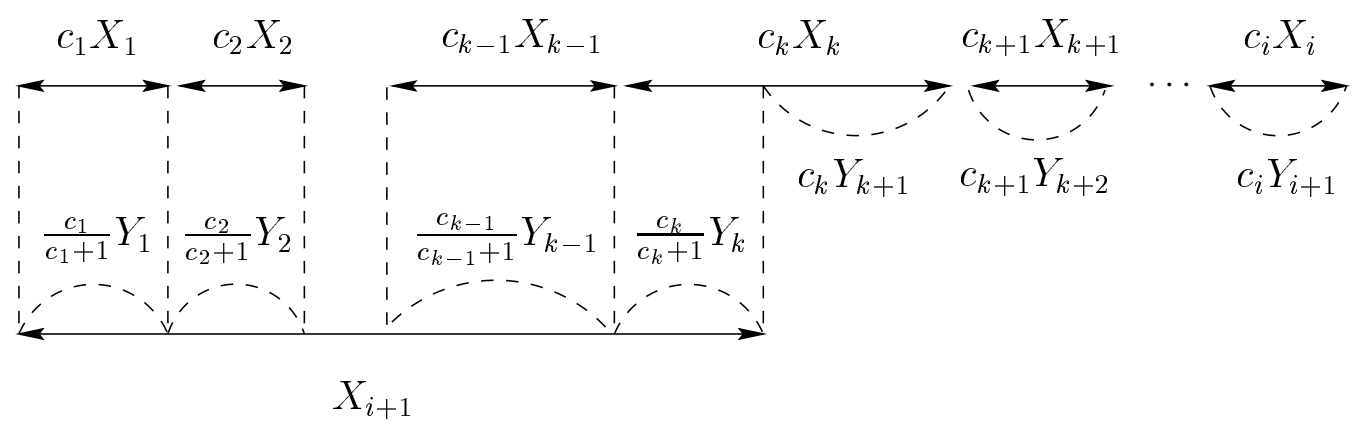

Figure 1: Coupling of the random variables $X_{1}, \ldots, X_{i+1}$ under event $E_{i+1, k}(\mathbf{c})$

exponential random variables $Y_{1}, Y_{2}, \ldots$ in the following way:

$$
\begin{aligned}
& X_{j}=\frac{1}{c_{j}+1} Y_{j}, \quad 1 \leq j \leq k-1 ; \quad X_{k}=\frac{1}{c_{k}+1} Y_{k}+Y_{k+1} ; \\
& X_{j}=Y_{j+1}, \quad k+1 \leq j \leq i ; \quad X_{i+1}=\sum_{j=1}^{k} \frac{c_{j}}{c_{j}+1} Y_{j} ; \\
& X_{j}=Y_{j}, \quad j \geq i+2 .
\end{aligned}
$$

It is easy to check that (8) is also applicable when $k=1$. Similarly, given an event $E_{i+1, i+1}(\mathbf{c})$ we have

$$
\begin{aligned}
& X_{j}=\frac{1}{c_{j}+1} Y_{j}, \quad 1 \leq j \leq i ; \quad X_{i+1}=\sum_{j=1}^{i} \frac{c_{j}}{c_{j}+1} Y_{j}+Y_{i+1} ; \\
& X_{j}=Y_{j}, \quad j \geq i+2 .
\end{aligned}
$$

Thus, given an event $E_{i+1, k}(\mathbf{c})$ for some $k=1,2, \ldots, i+1$ one can replace $X_{j}$ 's by $Y_{j}$ 's. We summarize our arguments in the following theorem.

Theorem 2.1 Let $f\left(x_{1}, x_{2}, \ldots\right)$ be an arbitrary function defined for $x_{1}, x_{2}, \ldots \geq 0$. Then for any $i \geq 1$ and any $\mathbf{c}$ defined by (7) the conditional distribution of $f\left(X_{1}, X_{2}, \ldots\right)$ given an event $E_{i+1, k}(\mathbf{c})$ for some $k=1,2, \ldots, i$ (or $\left.k=i+1\right)$ coincides with the unconditional distribution of the same function, where the arguments are replaced according to (8) (or according to (9)).

Now let us condition on $E_{i+1, k}(\mathbf{c})$, where $k=1,2, \ldots, i$, and see what happens to $S_{l}$ for some $l \geq i+1$. From (8) we have

$$
\begin{aligned}
S_{l} & =X_{1}+X_{2}+\ldots+X_{l} \\
& =\sum_{j=1}^{k} \frac{1}{c_{j}+1} Y_{j}+Y_{k+1}+\ldots+Y_{i+1}+\sum_{j=1}^{k} \frac{c_{j}}{c_{j}+1} Y_{j}+Y_{i+2}+\ldots+Y_{l} \\
& =Y_{1}+Y_{2}+\ldots+Y_{l} .
\end{aligned}
$$

The same holds, if we condition on $E_{i+1, i+1}(\mathbf{c})$ and apply (9). It is convenient to formulate this important property of (8), (9) in the following lemma. 
Lemma 2.2 For any $i \geq 1 ; k=1,2, \ldots, i+1$ and any c defined by (7) appropriate replacements (8) or (9) under event $E_{i+1, k}(\mathbf{c})$ provide

$$
S_{l}=Y_{1}+Y_{2}+\ldots+Y_{l}, \quad l \geq i+1
$$

In other words, conditioning on linear inequalities does not affect the structure of a sum. Lemma 2.2 has the following corollary, which is a counterintuitive property of exponential random variables.

Corollary 2.3 For any $t \in R$ and any $\mathbf{c}$ defined by (7) holds

$$
\operatorname{Pr}\left(S_{l}<t \mid E_{i+1, k}(\mathbf{c})\right)=\operatorname{Pr}\left(S_{l}<t\right), \quad 1 \leq k \leq i+1 \leq l .
$$

From Corollary 2.3 it follows for example that

$$
\operatorname{Pr}\left(X_{1}+X_{2}+X_{3}<t \mid X_{3}>1000 X_{1}+1000 X_{2}\right)=\operatorname{Pr}\left(X_{1}+X_{2}+X_{3}<t\right) .
$$

Remark 2.4 Note that Corollary 2.3 itself has simpler proofs. For example,

$$
\begin{aligned}
& \operatorname{Pr}\left(S_{3}<t \mid X_{3}>1000 X_{1}+1000 X_{2}\right) \\
& \quad=\operatorname{Pr}\left(S_{3}<t \mid X_{3} / S_{3}>1000\left(X_{1} / S_{3}\right)+1000\left(X_{2} / S_{3}\right)\right)=\operatorname{Pr}\left(S_{3}<t\right),
\end{aligned}
$$

where the last equality holds since $X_{1} / S_{3}, X_{2} / S_{3}$ and $X_{3} / S_{3}$ are independent of $S_{3}$.

\section{Two examples}

In this section we give two examples, where conditioning on linear inequalities allows obtaining a simple representation of quite complicated functions whose arguments are either i.i.d. exponentials or uniform spacings.

Our first example is a generalization of (3) and (4).

Theorem 3.1 For any $m=1,2, \ldots$ and any $c>1$ holds

$$
\sum_{i=1}^{m} \min \left\{(c-1) S_{i}, X_{i+1}\right\} \stackrel{d}{=} \sum_{i=1}^{m}\left(1-\frac{1}{c^{i}}\right) X_{i} .
$$

Uniform $(n+1)$-spacings, where $n \geq m$, satisfy

$$
\sum_{i=1}^{m} \min \left\{(c-1) \tilde{S}_{i}, D_{i+1}\right\} \stackrel{d}{=} \sum_{i=1}^{m}\left(1-\frac{1}{c^{i}}\right) D_{i} .
$$


Proof. We prove (10) by subsequently expanding $\min \left\{(c-1) S_{i}, X_{i+1}\right.$ for $i=1,2, \ldots, m$, and we perform the proof by induction with respect to $i$.

Assume that after expanding the first $i-1$ minima, where $i=1,2, \ldots m$, we have

$$
\sum_{i=1}^{m} \min \left\{(c-1) S_{i}, X_{i+1}\right\} \stackrel{d}{=} \sum_{j=1}^{i-1}\left(1-\frac{1}{c^{i-j}}\right) X_{j}+\sum_{j=i}^{m} \min \left\{(c-1) S_{j}, X_{j+1}\right\} .
$$

This trivially holds for $i=1$. We are going to show that if (12) is valid for $i$, then it is also valid for $i+1$. Considering the $i$ th minimum $\min \left\{S_{i}, X_{i+1}\right\}$ we use the arguments based on Theorem 2.1. Put $\mathbf{c}=(c-1, c-1, \ldots)$. Given an event

$$
E_{i+1, k}(\mathbf{c})=\left[(c-1) S_{k-1}<X_{i+1}<(c-1) S_{k}\right]
$$

for some $k=1,2, \ldots i$ we have, according to (8):

$$
\begin{array}{ll}
X_{j}=\frac{1}{c} Y_{j}, \quad 1 \leq j \leq k-1 ; & X_{k}=\frac{1}{c} Y_{k}+Y_{k+1} \\
X_{j}=Y_{j+1}, \quad k+1 \leq j \leq i ; & X_{i+1}=\sum_{j=1}^{k} \frac{c-1}{c} Y_{j} \\
X_{j}=Y_{j}, \quad j \geq i+2, &
\end{array}
$$

and

$$
\min \left\{(c-1) S_{i}, X_{i+1}\right\}=X_{i+1}=\sum_{j=1}^{k} \frac{c-1}{c} Y_{j}
$$

For the right-hand side of (12) conditioning on event $E_{i+1, k}(\mathbf{c})$ means replacing the $X_{j}$ 's by $Y_{j}$ 's. Straightforward calculations together with Lemma 2.2 give

$$
\sum_{j=1}^{i}\left(1-\frac{1}{c^{i+1-j}}\right) Y_{j}+\sum_{j=i+1}^{m} \min \left\{(c-1)\left(Y_{1}+Y_{2}+\ldots+Y_{j}\right), Y_{j+1}\right\} .
$$

Expression (13) does not depend on $k$. Moreover, along the same lines, but using (9) instead of (8), one can verify that given an event

$$
E_{i+1, i+1}(\mathbf{c})=\left[(c-1) S_{i}<X_{i+1}\right]
$$

the right-hand side of (12) again has the same distribution as (13). Now it immediately follows from (12) and the law of total probability that

$$
\sum_{i=1}^{m} \min \left\{(c-1) S_{i}, X_{i+1}\right\} \stackrel{d}{=} \sum_{j=1}^{i}\left(1-\frac{1}{c^{i+1-j}}\right) X_{j}+\sum_{j=i+1}^{m} \min \left\{(c-1) S_{j}, X_{j+1}\right\},
$$

where the $Y_{j}$ 's in (13) are again replaced by $X_{j}$ 's. 
Thus, by subsequently expanding all minima in the left-hand side of (10) we finally obtain

$$
\sum_{i=1}^{m} \min \left\{(c-1) S_{i}, X_{i+1}\right\} \stackrel{d}{=} \sum_{j=1}^{m}\left(1-\frac{1}{c^{m+1-j}}\right) X_{j},
$$

which is exactly (10).

One can show (see Remark 1.1) that formula (10) is equivalent to (11).

Remark 3.2 Note that (11) can also be proved along the same lines as (10). We have to only consider normalized exponentials $X_{1} / S_{n+1}, X_{2} / S_{n+1}, \ldots, X_{n+1} / S_{n+1}$ instead of just i.i.d. exponentials $X_{1}, X_{2}, \ldots$ and apply Lemma 2.2 to show that use of (8) or (9) in the proof would always provide

$$
S_{n+1}=Y_{1}+Y_{2}+\cdots+Y_{n+1}
$$

for $n \geq m$. Due to (2) it gives (11).

Remark 3.3 Putting $c=2, m=n$ in (11) we again derive formula (3). This result was proved earlier by Litvak and Adan [7], but it looked very exceptional, because it was not clear which feature of the left side of (3) was the crucial one. The proof of Theorem 3.1 nearly repeats the proof of (3) from Litvak and Adan [7], but now we see that the result is to a considerable extent based on Lemma 2.2. Namely, it is important that while expanding the $i$ th minimum $\min \left\{(c-1) S_{i}, X_{i+1}\right\}$ we keep the structure of the terms $\min \left\{(c-1) S_{j}, X_{j+1}\right\}, j \geq i+1$ for any $c>1$. That is why (4) and (3) allow generalizations (10) and (11).

The distribution $F_{m}(t)$ of the right side of (10) can be found through a fraction expansion of its Laplace-Stieltjes transform $\varphi_{m}(s)$ given by

$$
\varphi_{m}(s)=\prod_{i=1}^{m} \frac{c^{i}}{\left(c^{i}-1\right) s+c^{i}}=\sum_{i=1}^{m} \frac{c^{i}}{\left(c^{i}-1\right) s+c^{i}}\left(c^{i}-1\right)^{m-1} \prod_{\substack{j=1 \\ j \neq i}}^{m} \frac{c^{j}}{c^{i}-c^{j}} .
$$

Inversion of $\varphi_{m}(s)$ gives

$$
F_{m}(t)=\sum_{i=1}^{m}\left(c^{i}-1\right)^{m-1}\left(1-e^{-\frac{c^{i}}{c^{i}-1} t}\right) \prod_{\substack{j=1 \\ j \neq i}}^{m} \frac{c^{j}}{c^{i}-c^{j}}
$$

The moments can be found by integrating (14):

$$
\mathrm{E}\left(\sum_{i=1}^{m}\left(1-\frac{1}{c^{i}}\right) X_{i}\right)^{k}=k ! \sum_{i=1}^{m} \frac{\left(c^{i}-1\right)^{m+k-1}}{c^{k i}} \prod_{\substack{j=1 \\ j \neq i}}^{m} \frac{c^{j}}{c^{i}-c^{j}} .
$$


Further, we derive a distribution function $\tilde{F}_{m, n}(t)$ of the left side of (11) using Theorem 2 of Ali and Obaidullah [2], which actually has been proved in Ali [1]. For $m=n$ it gives

$$
\tilde{F}_{n, n}(t)=\sum_{i=0}^{n}\left(c^{i} t-c^{i}+1\right)_{+}^{n} \prod_{\substack{j=0 \\ j \neq i}}^{n} \frac{c^{j}}{c^{j}-c^{i}}=1-\sum_{i=0}^{n}\left(c^{i} t-c^{i}+1\right)_{-}^{n} \prod_{\substack{j=0 \\ j \neq i}}^{n} \frac{c^{j}}{c^{j}-c^{i}}, \quad 0 \leq t \leq 1
$$

where $x_{+}=x$, if $x>0$, and $x_{+}=0$ otherwise; $x_{-}=x$, if $x<0$, and $x_{-}=0$ otherwise. Putting $c=2$ we retrieve a distribution of the left side of (3) from Litvak and Adan [7]. The last equality above is valid since for any $t$ holds

$$
\sum_{i=0}^{n}\left(c^{i} t-c^{i}+1\right)^{n} \prod_{\substack{j=0 \\ j \neq i}}^{n} \frac{c^{j}}{c^{j}-c^{i}}=1
$$

A simple proof for the case $c=2$ can be found in Litvak and Adan [7].

The distribution function $\tilde{F}_{m, n}(t)$ for $m<n$ will be more complicated since the spacings in the right part of (11) are multiplied by coefficients which are no longer distinct. Namely, the spacings $D_{m+1}, D_{m+2}, \ldots, D_{n+1}$ do not participate in the sum, i.e. they actually have the same coefficient (zero). Nevertheless, there is still a relatively easy way to write $\tilde{F}_{m, n}(t)$ :

$$
\tilde{F}_{m, n}(t)=1-\sum_{i=1}^{m}\left(\frac{1}{1-c^{i}}\right)^{n-m+1}\left(c^{i} t-c^{i}+1\right)_{-}^{n} \prod_{\substack{j=1 \\ j \neq i}}^{m} \frac{c^{j}}{c^{j}-c^{i}}, \quad 0 \leq t \leq 1 .
$$

Now we find the moments of the right-hand side of (11):

$$
\begin{aligned}
\mathrm{E}\left(\sum_{i=1}^{m}\left(1-\frac{1}{c^{i}}\right) D_{i}\right)^{k} & =\sum_{\substack{k_{1}, k_{2}, \ldots, k_{m} \geq 0 \\
k_{1}+k_{2}+\cdots+k_{m}=k}} \frac{k !}{k_{1} ! k_{2} ! \ldots k_{m} !} \mathrm{E}\left(D_{1}^{k_{1}} D_{2}^{k_{2}} \ldots D_{m}^{k_{m}}\right) \prod_{i=1}^{m}\left(1-\frac{1}{c^{i}}\right)^{k_{i}} \\
& =\left(\begin{array}{c}
n+k \\
k
\end{array}\right)^{-1} \sum_{\substack{k_{1}, k_{2}, \ldots, k_{m} \geq 0 \\
k_{1}+k_{2}+\cdots, k_{m}=k}} \prod_{i=1}^{m}\left(1-\frac{1}{c^{i}}\right)^{k_{i}} .
\end{aligned}
$$

Here the last equality is provided by (cf. Sec. 13.1 in Karlin and Taylor [5])

$$
\mathrm{E}\left(D_{1}^{k_{1}} D_{2}^{k_{2}} \ldots D_{n+1}^{k_{n+1}}\right)=\frac{k_{1} ! k_{2} ! \ldots k_{n+1} ! n !}{\left(n+k_{1}+k_{2}+\cdots+k_{n+1}\right) !}, \quad k_{1}, k_{2}, \ldots, k_{n+1} \geq 0 .
$$

Particularly, we find

$$
\mathrm{E}\left(\sum_{i=1}^{n}\left(1-\frac{1}{c^{i}}\right) D_{i}\right)=\frac{n}{n+1}-\frac{1}{(n+1)(c-1)}\left(1-\frac{1}{c^{n}}\right) .
$$

Putting $c=2$ we again obtain a result from Litvak et al. [6].

Our second example is the following. 
Theorem 3.4 For any $m=2,3, \ldots$ and any $c>0$ holds

$$
\min _{1 \leq i \leq m}\left\{\sum_{j=1}^{i-1} c X_{j}+\sum_{j=i+1}^{m} X_{j}\right\} \stackrel{d}{=} \sum_{i=1}^{m-1}\left(1-\frac{c-1}{c^{i+1}-1}\right) X_{i} .
$$

Uniform $(n+1)$-spacings, where $n+1 \geq m$, satisfy

$$
\min _{1 \leq i \leq m}\left\{\sum_{j=1}^{i-1} c D_{j}+\sum_{j=i+1}^{m} D_{j}\right\} \stackrel{d}{=} \sum_{i=1}^{m-1}\left(1-\frac{c-1}{c^{i+1}-1}\right) D_{i} .
$$

Proof. We shall prove (16) again by induction with respect to $i$. Denote

$$
L_{i}=\sum_{j=1}^{i-1} c X_{j}+\sum_{j=i+1}^{m} X_{j}, \quad 1 \leq i \leq m .
$$

Assume that for some $i=1,2, \ldots, m-1$ holds

$$
\begin{aligned}
& \min \left\{L_{1}, L_{2}, \ldots, L_{m}\right\} \\
& \stackrel{d}{=} \min \left\{\sum_{j=1}^{i-1}\left(1-\frac{c-1}{c^{i-j+1}-1}\right) X_{j}+\sum_{j=i+1}^{m} X_{j}, L_{i+1}, L_{i+2}, \ldots, L_{m}\right\} .
\end{aligned}
$$

Equation (18) is trivial for $i=1$. It remains to show that if (18) is valid for $i$ than it is also valid for $i+1$. Note that

$$
\begin{aligned}
& \min \left\{\sum_{j=1}^{i-1}\left(1-\frac{c-1}{c^{i-j+1}-1}\right) X_{j}+\sum_{j=i+1}^{m} X_{j}, L_{i+1}\right\} \\
& =\sum_{j=1}^{i-1}\left(1-\frac{c-1}{c^{i-j+1}-1}\right) X_{j}+\sum_{j=i+2}^{m} X_{j}+\min \left\{X_{i+1}, \sum_{j=1}^{i} \frac{c^{i-j+1}(c-1)}{c^{i-j+1}-1} X_{j}\right\} .
\end{aligned}
$$

Consider events $E_{i+1, k}(\mathbf{c}), 1 \leq k \leq i+1$, where the first $i$ coordinates of vector $\mathbf{c}$ are defined by

$$
c_{j}=\frac{c^{i-j+1}(c-1)}{c^{i-j+1}-1}, \quad 1 \leq j \leq i .
$$

Given an event $E_{i+1, k}(\mathbf{c})$ for some $k=1,2, \ldots i$ we change $X_{j}$ 's to $Y_{j}$ 's according to (8):

$$
\begin{aligned}
& X_{j}=\frac{c^{i-j+1}-1}{c^{i-j+2}-1} Y_{j}, \quad 1 \leq j \leq k-1 ; \quad X_{k}=\frac{c^{i-k+1}-1}{c^{i-k+2}-1} Y_{k}+Y_{k+1} \\
& X_{j}=Y_{j+1}, \quad k+1 \leq j \leq i ; \quad X_{i+1}=\sum_{j=1}^{k} \frac{c^{i-j+1}(c-1)}{c^{i-j+2}-1} Y_{j} \\
& X_{j}=Y_{j}, \quad j \geq i+2 .
\end{aligned}
$$


Also, the event $E_{i+1, k}(\mathbf{c})$ provides

$$
\min \left\{X_{i+1}, \sum_{j=1}^{i} \frac{c^{i-j+1}(c-1)}{c^{i-j+1}-1} X_{j}\right\}=X_{i+1}=\sum_{j=1}^{k} \frac{c^{i-j+1}(c-1)}{c^{i-j+2}-1} Y_{j} .
$$

Now we replace the $X_{j}$ 's by $Y_{j}$ 's in the right-hand side of (19) yielding

$$
\sum_{j=1}^{i}\left(1-\frac{c-1}{c^{i-j+2}-1}\right) Y_{j}+\sum_{j=i+2}^{m} Y_{j}
$$

According to Theorem 2.1 expression (20) has the same distribution as the right-hand side of (19) conditioned on event $E_{i+1, k}(\mathbf{c})$. However, (20) does not depend on $k$. Furthermore, conditioning on event $E_{i+1, i+1}(\mathbf{c})$ we shall have the same expression. Also, it follows from Lemma 2.2 that changing $X_{j}$ 's to $Y_{j}$ 's by use of (8) or (9) doesn't affect the structure of $L_{i+2}, L_{i+3}, \ldots, L_{m}$. Hence,

$$
\begin{gathered}
\min \left\{\sum_{j=1}^{i-1}\left(1-\frac{c-1}{c^{i-j+1}-1}\right) X_{j}+\sum_{j=i+1}^{m} X_{j}, L_{i+1}, L_{i+2}, \ldots, L_{m}\right\} \\
\stackrel{d}{=} \min \left\{\sum_{j=1}^{i}\left(1-\frac{c-1}{c^{i-j+2}-1}\right) X_{j}+\sum_{j=i+2}^{m} X_{j}, L_{i+2}, \ldots, L_{m}\right\} .
\end{gathered}
$$

Here $Y_{j}$ 's are again replaced by $X_{j}$ 's.

Thus, subsequently reducing the set $\left\{L_{1}, L_{2}, \ldots, L_{m}\right\}$ we finally obtain

$$
\min \left\{L_{1}, L_{2}, \ldots, L_{m}\right\} \stackrel{d}{=} \sum_{j=1}^{m-1}\left(1-\frac{c-1}{c^{m-j+1}-1}\right) X_{j}
$$

which is exactly (16).

Equation (17) is equivalent to (16) (see Remark 1.1). Also, (17) can be proved separately along the same lines as (16) (see Remark 3.2).

As above, the distribution function $G_{m}(t)$ of the right-hand side of (16) can be obtained via its Laplace-Stieltjes transform:

$$
G_{m}(t)=\sum_{i=1}^{m-1}\left(\frac{c^{i}-1}{c-1}\right)^{m-2}\left(1-e^{-\frac{c^{i+1}-1}{c\left(c^{i}-1\right)} t}\right) \prod_{\substack{j=1 \\ j \neq i}}^{m-1} \frac{c^{j+1}-1}{c^{i}-c^{j}}
$$

The moments are

$$
\mathrm{E}\left(\sum_{i=1}^{m-1}\left(1-\frac{c-1}{c^{i+1}-1}\right) X_{i}\right)^{k}=k ! \sum_{i=1}^{m-1} \frac{c^{k}\left(c^{i}-1\right)^{m+k-2}}{(c-1)^{m-2}\left(c^{i+1}-1\right)^{k}} \prod_{\substack{j=1 \\ j \neq i}}^{m-1} \frac{c^{j+1}-1}{c^{i}-c^{j}}, \quad k \geq 1 .
$$




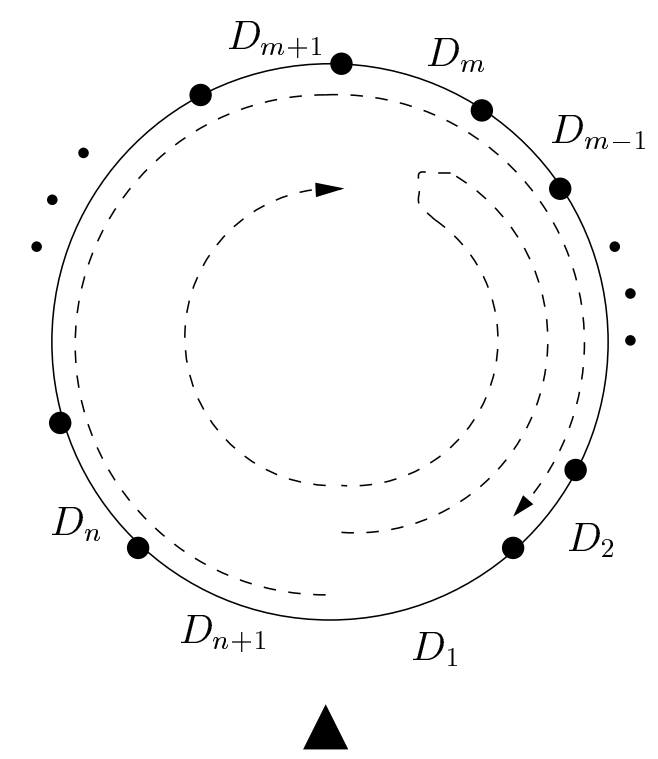

Figure 2: A carousel system.

The distribution function $\tilde{G}_{m, n}(t)$ of the right part of (17) can again be found by Theorem 2 of Ali and Obaidullah [2]. The easiest representation is

$$
\begin{aligned}
\tilde{G}_{m, n}(t) & =1-\sum_{i=1}^{m-1}\left(\frac{c-1}{c\left(1-c^{i}\right)}\right)^{n-m+2}\left(\frac{c^{i+1}-1}{c-1} t-\frac{c^{i+1}-1}{c-1}+1\right)_{-}^{n} \prod_{\substack{j=1 \\
j \neq i}}^{m-1} \frac{c^{j+1}-1}{c^{j+1}-c^{i+1}}, \\
0 \leq t \leq 1 . &
\end{aligned}
$$

Further, using (15) we obtain

$$
\mathrm{E}\left(\sum_{i=1}^{m-1}\left(1-\frac{c-1}{c^{i+1}-1}\right) D_{i}\right)^{k}=\left(\begin{array}{c}
n+k \\
k
\end{array}\right)^{-1} \sum_{\substack{k_{1}, k_{2}, \ldots, k_{m-1} \geq 0 \\
k_{1}+k_{2}+\cdots+k_{m-1}=k}} \prod_{i=1}^{m-1}\left(1-\frac{c-1}{c^{i+1}-1}\right)^{k_{i}} .
$$

\section{An application to carousel systems}

A carousel, or paternoster, is a computer controlled warehousing system which is widely used to store small and medium sized goods which are required moderately often. The system consists of a large number of shelves or drawers rotating in a closed loop in either direction. The picker has a fixed position in front of the carousel, which rotates the required items to the picker.

Following Bartoldi and Platzman [3] and Rouwenhorst et al. [10] we represent a carousel as a circle of length 1 (see Figure 2).

Let $U_{0}=0$ be the picker's starting point, and let $U_{i}$, where $i=1,2, \ldots, n$, be the position of the $i$ th item. In practice the items are often assigned to the positions in alphabetic order. Hence, it is reasonable to assume that random variables $U_{i}, i=1,2, \ldots, n$, are 
independent and uniformly distributed on $[0,1)$. The picker's starting point plus $n$ items partition the circle in $n+1$ uniform spacings $D_{1}, D_{2}, \ldots, D_{n+1}$ defined by (1).

The picker follows a certain route to collect all the items. It would be very natural for the picker to follow the Nearest Item (NI) heuristic, where the next item to pick is always the nearest one. This simple algorithm performs quite well, and it is frequently used in real warehouses. It was shown by Litvak et al. [6] that the travel time $T_{n}^{N I}$ under the NI heuristic satisfies

$$
T_{n}^{N I} \stackrel{d}{=} \sum_{i=1}^{n} \min \left\{\tilde{S}_{i}, D_{i+1}\right\}
$$

The distribution, the moments and the asymptotic properties of $T_{n}^{N I}$ were obtained in Litvak and Adan [7] as consequences of (3). As we saw in the previous section, formula (3) is just a special case of (11).

We can also give an interpretation for the right-hand side of (17) via the travel time in a carousel system. Let $m=1,2, \ldots, n$. Suppose that the picker is allowed to perform at most $m-1$ steps counterclockwise, and after that he must collect the rest of the items clockwise. Then $m$ possible routes are:

$$
\begin{aligned}
& D_{n+1}+D_{n}+\cdots+D_{2}, \\
& D_{n+1}+D_{n}+\cdots+D_{3}+2 D_{1}, \\
& \cdots, \\
& D_{n+1}+D_{n}+\cdots+D_{m}+2 D_{m-2}+2 D_{m-3}+\cdots+2 D_{1}, \\
& D_{n+1}+D_{n}+\cdots+D_{m+1}+2 D_{m-1}+2 D_{m-2}+\cdots+2 D_{1} .
\end{aligned}
$$

The first and the last of those routes are shown on Figure 2 with dashed lines. The shortest route would be

$$
\begin{aligned}
T_{n}^{(m)} & =D_{n+1}+D_{n}+\cdots+D_{m+1}+\min _{1 \leq i \leq m}\left\{\sum_{j=1}^{i-1} 2 D_{j}+\sum_{j=i+1}^{m} D_{j}\right\} \\
& \stackrel{d}{=} D_{n+1}+D_{n}+\cdots+D_{m+1}+\sum_{i=1}^{m-1}\left(1-\frac{1}{2^{i+1}-1}\right) D_{i},
\end{aligned}
$$

where the last equality follows from (17). Now by Ali and Obaidullah [2] the distribution function of $T_{n}^{(m)}$ is

$$
\begin{aligned}
& \operatorname{Pr}\left(T_{n}^{(m)}<t\right)=\sum_{i=0}^{m-1}\left(\left(2^{i+1}-1\right) t-2^{i+1}+2\right)_{+}^{n} \prod_{\substack{j=0 \\
j \neq i}}^{m-1} \frac{2^{j+1}-1}{2^{j+1}-2^{i+1}}, \quad 0 \leq t \leq 1 ; \\
& \operatorname{Pr}\left(T_{n}^{(m)}<t\right)=1, \quad t>1 .
\end{aligned}
$$

The moments can be obtained directly from (22):

$$
\mathrm{E}\left(T_{n}^{(m)}\right)^{k}=\left(\begin{array}{c}
n+k \\
k
\end{array}\right)^{-1} \sum_{\substack{k_{1}, k_{2}, \ldots, k_{n} \geq 0 \\
k_{1}+k_{2}+\cdots+k_{n}=k}} \prod_{i=1}^{m-1}\left(1-\frac{1}{2^{i+1}-1}\right)^{k_{i}} .
$$


It is clear that

$$
T_{n}^{(n)} \leq T_{n}^{(n-1)} \leq \cdots \leq T_{n}^{(1)} .
$$

Let us give a more detailed interpretation for $T_{n}^{(n)}$. Suppose that the picker must perform the last step clockwise, and he has to choose the best route of this kind. We shall call this route a One-Side Optimal (OSO) route. Note that it is never good to cover the same interval more than twice. Hence, the picker will choose a route with at most one turn. It means that he can either pick all the items clockwise or he can make several steps counterclockwise and then turn. Since the last step must be performed clockwise, the number of steps performed counterclockwise can not exceed $n-1$. Thus, the travel time $T_{n}^{O S O}$ for the OSO route is actually $T_{n}^{(n)}$. We know from (22) that

$$
T_{n}^{O S O}=D_{n+1}+\min _{1 \leq i \leq n}\left\{\sum_{j=1}^{i-1} 2 D_{j}+\sum_{j=i+1}^{n} D_{j}\right\} \stackrel{d}{=} D_{n+1}+\sum_{i=1}^{n-1}\left(1-\frac{1}{2^{i+1}-1}\right) D_{i} .
$$

Now we can compare the distributions of the travel times under the OSO route and the NI heuristic. It follows from above that both $T_{n}^{N I}$ and $T_{n}^{O S O}$ are distributed as a sum of $n$ spacings which are multiplied by certain coefficients. The coefficients are

$$
\begin{array}{ccc}
T_{n}^{N I}: & \frac{1}{2}, \frac{3}{4}, \ldots, \quad 1-\frac{1}{2^{n-1}}, \quad 1-\frac{1}{2^{n}} \\
T_{n}^{O S O}: & \frac{2}{3}, \frac{6}{7}, \ldots, 1-\frac{1}{2^{n}-1}, & 1 .
\end{array}
$$

It is clearly seen that the travel time under the NI heuristic is stochastically smaller than the travel time under the OSO route, i.e.

$$
\operatorname{Pr}\left(T_{n}^{N I}<t\right) \geq \operatorname{Pr}\left(T_{n}^{O S O}<t\right), \quad t \geq 0, \quad n \geq 1 .
$$

Below we explain this result.

Consider a sum

$$
D_{2}+D_{3}+\cdots+D_{n+1}
$$

Suppose that a term $D_{i+1}$ for any $i=1,2, \ldots, n$ can be replaced by $\tilde{S}_{i}$. Let us say that $\alpha_{i}=1$, if such a replacement took place. Otherwise, we put $\alpha_{i}=0$. Denote

$$
\alpha=\left(\alpha_{1}, \alpha_{2}, \ldots, \alpha_{n}\right) \in\{0,1\}^{n} \text {. }
$$

After the replacements sum (24) becomes

$$
T_{n}(\alpha)=\sum_{i=1}^{n}\left[\left(1-\alpha_{i}\right) D_{i+1}+\alpha_{i} \tilde{S}_{i}\right]
$$

One can see that

$$
\sum_{i=1}^{n} \min \left\{\tilde{S}_{i}, D_{i+1}\right\}=\min _{\alpha \in\{0,1\}^{n}}\left\{T_{n}(\alpha)\right\}
$$


Roughly speaking, a random value $\sum_{i=1}^{n} \min \left\{\tilde{S}_{i}, D_{i+1}\right\}$ (remember that according to (21) it has the same distribution as $T_{n}^{N I}$ ) is a minimum of all random values that one can get from (24) replacing $D_{i+1}$ 's by $\tilde{S}_{i}$ 's. Indeed, assume that two vectors

$$
\alpha^{(1)}=\left(\alpha_{1}^{(1)}, \alpha_{2}, \ldots, \alpha_{n}\right) \text { and } \alpha^{(2)}=\left(\alpha_{1}^{(2)}, \alpha_{2}, \ldots, \alpha_{n}\right)
$$

differ only in their first coordinate. Such a pair of vectors exists in $\{0,1\}^{n}$ for any $\left(\alpha_{2}, \ldots, \alpha_{n}\right)$, and it is obvious that

$$
\min \left\{T_{n}\left(\alpha^{(1)}\right), T_{n}\left(\alpha^{(2)}\right)\right\}=\min \left\{D_{1}, D_{2}\right\}+\sum_{i=2}^{n}\left[\left(1-\alpha_{i}\right) D_{i+1}+\alpha_{i} \tilde{S}_{i}\right] .
$$

The whole set $\{0,1\}^{n}$ can be seen as a set consisting of $2^{n-1}$ pairs of vectors which differ only in their first coordinate. Hence,

$$
\min _{\alpha \in\{0,1\}^{n}}\left\{T_{n}(\alpha)\right\}=\min \left\{D_{1}, D_{2}\right\}+\min _{\left(\alpha_{2}, \alpha_{3}, \ldots, \alpha_{n}\right) \in\{0,1\}^{n-1}}\left\{\sum_{i=2}^{n}\left[\left(1-\alpha_{i}\right) D_{i+1}+\alpha_{i} \tilde{S}_{i}\right]\right\} .
$$

Further we consider pairs in $\{0,1\}^{n-1}$ such that a vector $\left(\alpha_{2}, \alpha_{3}, \ldots, \alpha_{n}\right)$ differs from the other vector in a pair only in a coordinate $\alpha_{2}$. Proceeding in this way, we obtain the desired observation.

Now let $\mathbf{A} \subset\{0,1\}^{n}$ be a set of $n$ vectors such that one of their coordinates is 1 and the other coordinates are 0 , i.e. exactly one of $D_{i+1}$ 's from $(24)$ is replaced by $\tilde{S}_{i}$. From (23) by changing indices we have

$$
T_{n}^{O S O} \stackrel{d}{=} D_{1}+\min _{2 \leq i \leq n+1}\left\{\sum_{j=2}^{i-1} 2 D_{j}+\sum_{j=i+1}^{n+1} D_{j}\right\}=\min _{\alpha \in \mathbf{A}}\left\{T_{n}(\alpha)\right\},
$$

where the last equality can be straightforwardly verified. Clearly,

$$
\min _{\alpha \in\{0,1\}^{n}}\left\{T_{n}(\alpha)\right\} \leq \min _{\alpha \in \mathbf{A}}\left\{T_{n}(\alpha)\right\}
$$

Thus, even before proving Theorem 3.1 and Theorem 3.4, already from (21), (25) and (26) one can see that the random value $T_{n}^{N I}$ would be stochastically smaller than $T_{n}^{O S O}$.

Remark 4.1 Note that in the discussion above we compare the NI heuristic and the OSO route only in distribution. It does not at all follow from our arguments that the NI heuristic always performs better than the OSO route. For example, assume that $n=5$, and the items to be picked are located at the positions 1/32, 3/32, 7/32, 15/32 and 30/32 (see Figure 3). The travel time under the NI heuristic is

$$
\frac{1}{32}+\frac{2}{32}+\frac{4}{32}+\frac{8}{32}+\frac{15}{32}=\frac{30}{32} \text {. }
$$

On the other hand, the OSO route provides a sequence $30 / 32,1 / 32,3 / 32,7 / 32,15 / 32$. The travel time of this route is

$$
\frac{2}{32}+\frac{2}{32}+\frac{1}{32}+\frac{2}{32}+\frac{4}{32}+\frac{8}{32}=\frac{19}{32}
$$




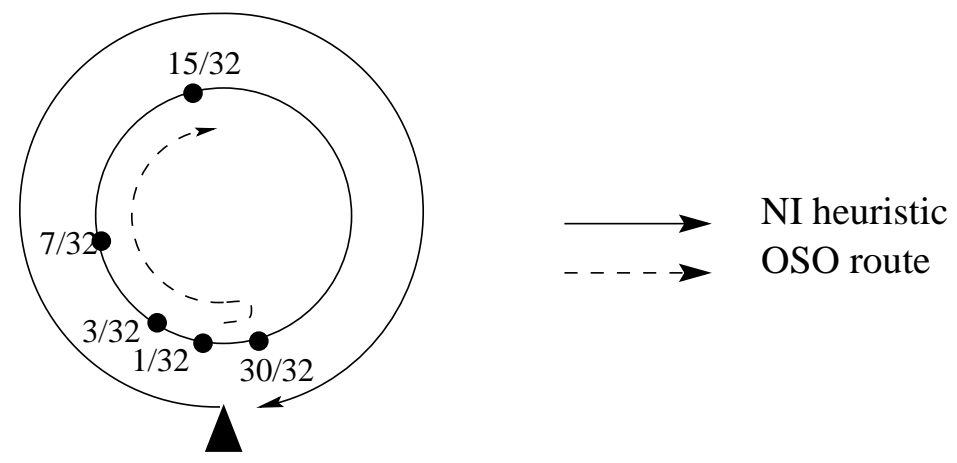

Figure 3: An example where the travel time for the OSO route is smaller than under the NI heuristic.

\section{Acknowledgement}

The author is grateful to Ivo Adan for fruitful collaboration. Also, the author would like to thank John Einmahl for a counterexample in Remark 1.1.

\section{References}

[1] M.M. Ali. Content of the frustum of a simplex. Pacific Journal of Math. 48(2), 313-322, 1973.

[2] M.M. Ali and M. Obaidullah. Distribution of linear combination of exponential variates. Commun. Statist.-Theor. Meth. 11(13), 1453-1463, 1982.

[3] J.J. B ARTOldi, III And L.K. Platzman. Retrieval strategies for a carousel conveyor. IIE Transactions, 18(2), 166-173, 1986.

[4] W. FELler. An introduction to a probabillity theory and its applications. Wiley, London, 1970.

[5] S. Karlin, H.M. Taylor. A second course in stochastic processes. Academic Press, London, 1981.

[6] N. Litvak, I. Adan, J. Wessels, and W.H.M. Zijm. Order picking in carousel systems under the nearest item heuristic. To appear in Prob. Engineer. Inform. Sci., 2001.

[7] N. LitvaK AND I. AdAn. The travel time in carousel systems under the nearest item heuristic. EURANDOM Technical Report 2000-016, Eindhoven, 2000.

[8] R. Pyke. Spacings. J. Roy. Statist. Soc. B. 27, 395-436, 1965.

[9] R. Pyke. Spacings revisited. In 6th Berkeley Symp., Math. Statist. Prob. 1, 417-427, 1972. 
[10] B. Rouwenhorst, J.P. van den Berg, G.J. van Houtum, and W.H.M. Zijm. Performance analysis of a carousel system. In Progress in Material Handling Research: 1996, The Material Handling Industry of America, Charlotte, NC, 495-511, 1996. 